\title{
Effect of material properties on optimal radial strain gage locations in sharp V-notched configurations
}

\author{
Pranjol Paul ${ }^{1, *}$, K.S.R.Krishna Murthy ${ }^{2}$, and Debabrata Chakraborty ${ }^{3}$ \\ ${ }^{1}$ Research scholar, Department of Mechanical Engineering, Indian Institute of Technology Guwahati, Guwahati - 781039, India \\ ${ }^{2,3}$ Department of Mechanical Engineering, Indian Institute of Technology Guwahati, Guwahati - 781039, India
}

\begin{abstract}
A simple yet reliable and powerful methodology using only one strain gage has been recommended for appropriate determination of notch stress intensity factor (NSIF) for sharp V-notched configurations subjected to mode I condition. The methodology is supported by strong theoretical postulates, and it permits the gage to be pasted prominently apart from tip of the notch thus avoiding various problems associated with singularities. Unlike the conventional methodologies, the recommended strain gage methodology also proposes optimal radial strain gage locations which are beneficial in appropriate determination of NSIFs. A FEM based numerical approach is adopted for obtaining optimal radial gage locations a priori for the aforesaid configuration. The optimal radial gage locations are observed to be influenced by parameters viz. the notch angle, the ratio of notch length to width of the plate and also material properties. Results were already published by the authors to establish that the optimal radial gage locations are influenced by the notch angle and the ratio of notch length to width of the plate. In this conference paper, a case is studied with a completely different material to check whether material properties influence the graphical trends of results or not.
\end{abstract}

\section{Introduction}

In engineering components, we may often come across stress raisers such as sharp V-notches, rounded tip Vnotches and re-entrant corners. In some cases, notches are intentionally introduced in the mechanical parts such as gears, screws, bolts and nuts in order to achieve defined engineering objectives. In addition, notches also exist as a result of manufacturing processes such as anisotropic chemical etching while fabrication of microelectromechanical components [1] and injection moldings for manufacturing polystyrene products [2].

A sharp V-notch is a V-notch which has a tip radius significantly smaller than the dimension of its sides. Supported by the eigen function expansion method, Williams [3] proved that singularity of stress occurs at the tip of a sharp V-notch as $\sigma_{i j}=K^{V} r^{\lambda^{\prime}-1} f_{i j}(\theta)$ where the order of the singularity $\lambda^{\prime}-1$ and the angular functions $f_{i j}(\theta)$ are functions of the notch angle $\gamma$, and $K^{V}$ is the notch stress intensity factor (NSIF). For a given set of geometrical and loading conditions, $K^{V}$ illustrates the state of the stress in the vicinity of the notch tip identical to the stress intensity factor (SIF) of cracked configurations in linear elastic fracture mechanics (LEFM). It is dependent on the geometry of the body and boundary conditions.

In case of engineering components made of brittle and quasi-brittle materials, the static and fatigue load carrying capacities reduce due to the occurrence of sharp V-notches. Several researches had been attempted to recommend the brittle fracture criterion at the sharp Vnotches [4-12]. Because of widespread use of $K^{V}$, significant efforts had been put in the development of several numerical [13-16] and experimental [17-22] methods for determination of NSIFs for different notched configurations.
Several experimental techniques such as strain gage [17, 18], photoelastic [19], caustics [20], coherent gradient sensing [21] and digital image correlation [22] had been developed to determine NSIFs under mode I and mixed mode conditions.

In a recently published research article, the authors (Paul et al. [23]) have postulated a novel and robust methodology in the domain of LEFM to determine the NSIF of sharp V-notched configurations by employing a technique using only one strain gage. The work [23] aimed to propose a novel strain gage technique based on the multiparameter strain series for appropriate determination of mode I NSIF $\left(K_{I}^{V}\right)$ of sharp Vnotched configurations made of isotropic materials. The proposed technique requires only one strain gage for the determination of $K_{I}^{V}$ which ought to be pasted apart from the notch-tip. Another aim of the study is to develop a methodology (supported by theoretical foundations) that reflects the occurence of a valid or optimal radial gage location and allows prior estimation of such optimal location for appropriate determination of $K_{I}^{V}$. The analysis [23] also aimed at relating the optimal radial gage locations with the geometry of the sharp Vnotched configuration.

Paul et al. also targets at relating the valid or optimal radial gage locations with the material properties and to confirm this observation a study similar to [23] is accomplished with a different material and the results are suitably shown in the successive section.

\section{Theoretical background}

The mathematical formulation of the proposed single strain gage technique and the theoretical foundation for obtaining optimal radial strain gage locations for 
appropriate determination of NSIF are briefed in this section. Illustration of these are available in the research article published by Paul et al. [23].

\subsection{Strain analysis of sharp V-notches}

The entire work is accomplished with an assumption of an isotropic and homogenous arbitrary two dimensional body with a sharp V-notch under mode I loading and plane stress conditions as shown in Fig. 1.

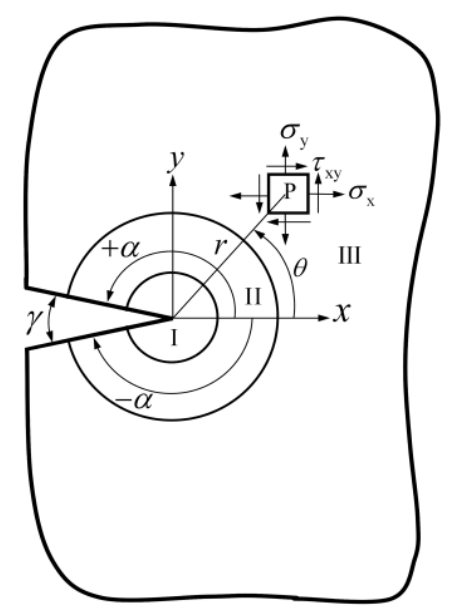

Fig.1. Typical zones (I, II and III) around the notch tip of a single edge notched tensile (SENT) configuration

Assuming stress-free notch surface boundary conditions ( $\sigma_{\theta}=\tau_{r \theta}=0$ at $\theta= \pm \alpha$, as shown in Fig. 1), it can be deduced that the stress components in polar coordinates under mode I loading condition is given by

$\left\{\begin{array}{c}\sigma_{r} \\ \sigma_{\theta} \\ \tau_{r \theta}\end{array}\right\}=\sum_{n=1}^{\infty} \operatorname{Re}\left\{\frac{\lambda_{n}^{\prime} A_{n}^{\prime}}{r^{1-\lambda_{n}^{\prime}}}\left[\begin{array}{c}\left(3-\lambda_{n}^{\prime}\right) \cos \left(\lambda_{n}^{\prime}-1\right) \theta+\left(\lambda_{n}^{\prime} \cos 2 \alpha+\cos 2 \lambda_{n}^{\prime} \alpha\right) \cos \left(\lambda_{n}^{\prime}+1\right) \theta \\ \left(\lambda_{n}^{\prime}+1\right) \cos \left(\lambda_{n}^{\prime}-1\right) \theta-\left(\lambda_{n}^{\prime} \cos 2 \alpha+\cos 2 \lambda_{n}^{\prime} \alpha\right) \cos \left(\lambda_{n}^{\prime}+1\right) \theta \\ \left(\lambda_{n}^{\prime}-1\right) \sin \left(\lambda_{n}^{\prime}-1\right) \theta-\left(\lambda_{n}^{\prime} \cos 2 \alpha+\cos 2 \lambda_{n}^{\prime} \alpha\right) \sin \left(\lambda_{n}^{\prime}+1\right) \theta\end{array}\right]\right\}$

where $n$ is the order of the term in the infinite series, $\lambda_{n}^{\prime}$ is eigenvalue under mode I condition and $A_{n}^{\prime}$ is coefficient of the Williams eigen function expansion. The satisfaction of the aforementioned boundary conditions also results into a characteristic equation for the solution of the eigenvalue $\lambda_{n}^{\prime}$ as

$$
\lambda_{n}^{\prime} \sin 2 \alpha+\sin 2 \lambda_{n}^{\prime} \alpha=0
$$

The positive root of the Eq. (2), $\lambda_{n}^{\prime}$, represents the order of singularity ahead of the notch tip under mode I loading condition which is influenced by the value of $\alpha$ and hence it is also influenced by the notch angle $\gamma$ (Fig. 1). Usually numerical methods are applied to solve the Eq. (2). $\lambda_{n}^{\prime}$ can be a real or a complex number which is influenced by the magnitude of the notch angle and can be expressed as

$$
\lambda_{n}^{\prime}=\lambda_{n}+i \lambda_{n}^{*}
$$

The first eigenvalue $\lambda_{1}^{\prime}$ is always real (i.e., $\lambda_{1}^{\prime}=\lambda_{1}$ and $\left.\lambda_{1}^{*}=0\right)$ and less than unity till $\gamma<180^{\circ}$ that represents singular stress behavior, and $\gamma=180^{\circ}$ represents a notchless solid i.e., $1-\lambda_{1}=0$ in Eq. (2). The second and higher order values of $\lambda_{n}^{\prime}$ will be real or complex numbers according to the notch angle. As the stresses should be real always, the Williams expansion coefficients $A_{n}^{\prime}$ can be either real or complex numbers according to the notch eigenvalues. Description about the nature of $\lambda_{n}^{\prime}$ and $A_{n}^{\prime}$ are available in Ref. [23]. Hence, the Williams coefficients $A_{n}^{\prime}$ can also be expressed as

$$
A_{n}^{\prime}=A_{n}+i A_{n}^{*}
$$

For $n=1$, the coefficient $A_{1}^{\prime}=A_{1} \quad\left(A_{1}^{*}=0\right.$ because $\lambda_{1}^{*}=0$ for $n=1$ ) represents singular term and is related to the NSIF $K_{I}^{V}$ as follows

$$
K_{I}^{V}=\lim _{r \rightarrow 0}\left(\sqrt{2 \pi} r^{1-\lambda_{1}} \sigma_{y}(\theta=0)\right)=\sqrt{2 \pi} \lambda_{1}\left(1+\lambda_{1}-\lambda_{1} \cos 2 \alpha-\cos 2 \alpha \lambda_{1}\right) A_{1}
$$

For $n>1, A_{n}^{\prime}$ are non-singular higher order coefficients. Using the stress transformation law, the stress components in Cartesian coordinate system (Fig.1) can be obtained under mode I loading as [23]

$\left\{\begin{array}{c}\sigma_{x} \\ \sigma_{y} \\ \tau_{x y}\end{array}\right\}=\sum_{n=1}^{\infty} \operatorname{Re}\left\{\frac{\lambda_{n}^{\prime} A_{n}^{\prime}}{r^{1-\lambda_{n}^{\prime}}}\left[\begin{array}{c}\left(2+\lambda_{n}^{\prime} \cos 2 \alpha+\cos 2 \alpha \lambda_{n}^{\prime}\right) \cos \left(\lambda_{n}^{\prime}-1\right) \theta-\left(\lambda_{n}^{\prime}-1\right) \cos \left(\lambda_{n}^{\prime}-3\right) \theta \\ \left(2-\lambda_{n}^{\prime} \cos 2 \alpha-\cos 2 \alpha \lambda_{n}^{\prime}\right) \cos \left(\lambda_{n}^{\prime}-1\right) \theta+\left(\lambda_{n}^{\prime}-1\right) \cos \left(\lambda_{n}^{\prime}-3\right) \theta \\ -\left(\lambda_{n}^{\prime} \cos 2 \alpha+\cos 2 \alpha \lambda_{n}^{\prime}\right) \sin \left(\lambda_{n}^{\prime}-1\right) \theta+\left(\lambda_{n}^{\prime}-1\right) \sin \left(\lambda_{n}^{\prime}-3\right) \theta\end{array}\right]\right\}$

Considering plane stress condition and utilizing the constitutive relationship, the strain components at a point $P(r, \theta)$ are given by

$$
\begin{aligned}
2 G \varepsilon_{x} & =\sum_{n=1}^{\infty} \operatorname{Re}\left\{\frac{\lambda_{n}^{\prime} A_{n}^{\prime}}{r^{1-\lambda_{n}^{\prime}}}\left[\begin{array}{l}
2 \kappa \cos \left(\lambda_{n}^{\prime}-1\right) \theta+\lambda_{n}^{\prime} \cdot \cos 2 \alpha \cdot \cos \left(\lambda_{n}^{\prime}-1\right) \theta+ \\
\cos 2 \lambda_{n}^{\prime} \alpha \cdot \cos \left(\lambda_{n}^{\prime}-1\right) \theta-\left(\lambda_{n}^{\prime}-1\right) \cdot \cos \left(\lambda_{n}^{\prime}-3\right) \theta
\end{array}\right]\right\} \\
2 G \varepsilon_{y} & =\sum_{n=1}^{\infty} \operatorname{Re}\left\{\frac{\lambda_{n}^{\prime} A_{n}^{\prime}}{r^{1-\lambda_{n}^{\prime}}}\left[\begin{array}{l}
2 \kappa \cos \left(\lambda_{n}^{\prime}-1\right) \theta-\lambda_{n}^{\prime} \cdot \cos 2 \alpha \cdot \cos \left(\lambda_{n}^{\prime}-1\right) \theta- \\
\cos 2 \lambda_{n}^{\prime} \alpha \cdot \cos \left(\lambda_{n}^{\prime}-1\right) \theta+\left(\lambda_{n}^{\prime}-1\right) \cdot \cos \left(\lambda_{n}^{\prime}-3\right) \theta
\end{array}\right]\right\} \\
2 G \gamma_{x y} & =\sum_{n=1}^{\infty} \operatorname{Re}\left\{\frac{\lambda_{n}^{\prime} A_{n}^{\prime}}{r^{1-\lambda_{n}^{\prime}}}\left[\begin{array}{l}
2\left(\lambda_{n}^{\prime}-1\right) \cdot \sin \left(\lambda_{n}^{\prime}-3\right) \theta- \\
2\left(\lambda_{n}^{\prime} \cos 2 \alpha+\cos 2 \lambda_{n}^{\prime} \alpha\right) \cdot \sin \left(\lambda_{n}^{\prime}-1\right) \theta
\end{array}\right]\right\}
\end{aligned}
$$

where $\kappa=(1-v) /(1+v)$ for plane stress condition and $G$ is the rigidity modulus. The strain series in Eq. (7) is an infinite series.

\subsection{Development of single strain gage technique}

For measuring surface strains with the help of strain gages, it is essential to locate a suitable zone around the notch tip. Zone I which is basically a small zone around the notch tip (Fig.1) is not appropriate for measurement of strains owing to high strain gradients and 3D state of 
stress. As the solutions of notch problems do exist in the form of infinite series, zone III which is the farthest zone is also not appropriate for the strain measurements. This is due to the fact that a large number of unknown coefficients needs to be included in the strain series for representing the strains at those remote locations and it also needs large number of strain gages. In addition, singular field may not exist at such distances. Therefore, the intermediate zone or zone II is an appropriate region for strain measurements. Here, it is presumed that strains in zone II contain the first term (singular term) of the Williams infinite series [23] and a few non-singular higher order terms. In the present investigation, zone II is assumed to be the region around the notch tip in which strains can be appropriately represented by the multiparameter strain series as shown in Eq. (7). The strains are comprised of a singular term and two nonsingular higher order terms when the infinite strain series is considered up to $n=2$ as per Ref. [23].

From Fig. 2, the normal strain component along the direction $a$ that makes an angle $\beta$ with the notch axis at a point $P$ can be obtained using strain transformation as

$$
\begin{aligned}
2 G \varepsilon_{a}= & r^{\lambda_{1}-1} A_{1} f_{1}\left(\theta, \beta, \lambda_{1}, \nu, \gamma\right)+r^{\lambda_{2}-1} A_{2} f_{2}\left(\theta, \beta, \lambda_{2}, \lambda_{2}^{*}, \gamma, \nu, r\right)+ \\
& r^{\lambda_{2}-1} A_{2}^{*} f_{2}^{*}\left(\theta, \beta, \lambda_{2}, \lambda_{2}^{*}, \gamma, \nu, r\right)
\end{aligned}
$$

where $\varepsilon_{a}$ is the normal strain along the longitudinal direction of strain gage, the functions $f_{1}, f_{2}$ and $f_{2}^{*}$ are the functions corresponding to the first order and the second order terms of the infinite strain series. The first order term of the infinite strain series always comprises real eigen value and thus real Williams coefficient of expansion. On the contrary, the second order term may comprise complex eigen value and thus complex Williams coefficient of expansion according to the magnitude of the notch angle as mentioned in preceding sections. Thus, in case of notched configurations leading to complex eigen value and Williams coefficient, the functions $f_{2}$ and $f_{2}^{*}$ will co-exist. However, in case of the notched configurations leading to real eigen value and Williams coefficient, the function $f_{2}$ will exist alone and the function $f_{2}^{*}$ will not exist. More in details would be discussed in successive paragraphs.

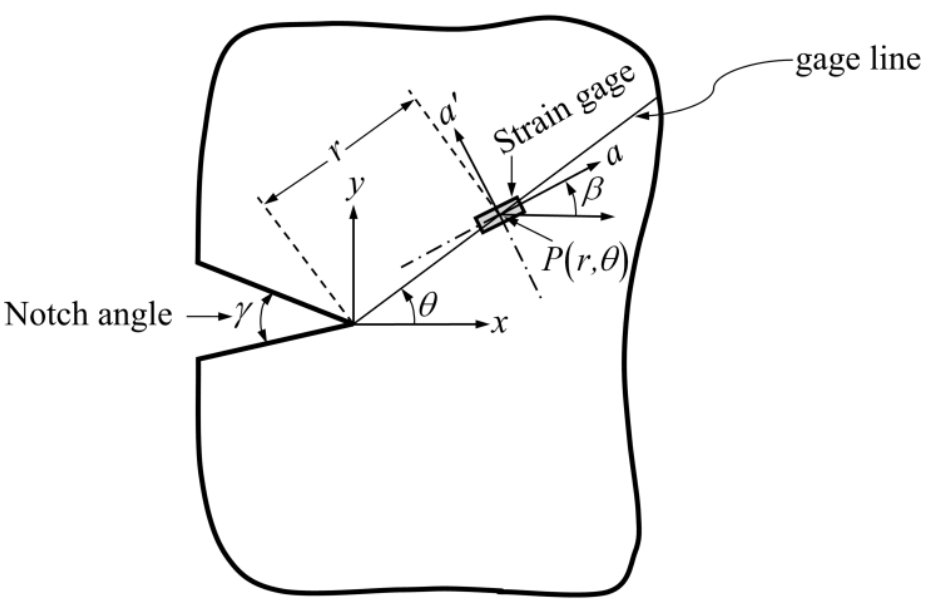

Fig.2. Schematic diagram depicting the orientation of strain gage in a sharp V-notched configuration

If $A_{1}$ is computed using Eq.(8), then the mode I SIF $K_{I}^{V}$ can subsequently be determined using Eq. (5). Straightaway one can apply three strain gages against three unknown coefficients $A_{1}, A_{2}$ and $A_{2}^{*}$ to yield three simultaneous equations using Eq.(8) to solve for $A_{1}, A_{2}$ and $A_{2}^{*}$ and resultantly the mode I SIF $K_{I}^{V}$. However, a meticulous manipulation has been accomplished to reduce the number of strain gages to only one which is the novelty of the published work in Ref.[23]. This methodology has significantly minimised the effort and also it is understood to be cost-effective in case of experimental work in similar domain. Finally, Eq. (8) could be reduced to the following equation

$$
2 G \varepsilon_{a}=r^{\lambda_{1}-1} A_{1} f_{1}\left(\theta, \beta, \lambda_{1}, v, \gamma\right)
$$

where

$$
f_{1}\left(\theta, \beta, \lambda_{1}, \nu, \gamma\right)=\lambda_{1}\left\{\begin{array}{l}
\cos ^{2} \beta\left[\begin{array}{l}
\cos \theta\left(\lambda_{1}-3\right)+\cos \theta\left(\lambda_{1}-1\right) \\
{\left[2 \kappa+\cos \lambda_{1}(2 \pi-\gamma)\right]}
\end{array}\right]- \\
\cos \theta\left(\lambda_{1}-3\right) \sin ^{2} \beta+ \\
2 \kappa \cos \theta\left(\lambda_{1}-1\right) \sin ^{2} \beta- \\
\cos \theta\left(\lambda_{1}-1\right) \cos \lambda_{1}(2 \pi-\gamma) \sin ^{2} \beta- \\
\sin \theta\left(\lambda_{1}-3\right) \sin 2 \beta- \\
\cos \lambda_{1}(2 \pi-\gamma) \sin \theta\left(\lambda_{1}-1\right) \sin 2 \beta- \\
\lambda_{1} \cos \beta \cos \theta\left(\lambda_{1}-3\right)+ \\
\lambda_{1} \cos { }^{2} \beta \cos \gamma \cos \theta\left(\lambda_{1}-1\right)+ \\
\lambda_{1} \cos \theta\left(\lambda_{1}-3\right) \sin { }^{2} \beta- \\
\lambda_{1} \cos \gamma \cos \theta\left(\lambda_{1}-1\right) \sin { }^{2} \beta+ \\
\lambda_{1} \sin \theta\left(\lambda_{1}-3\right) \sin 2 \beta- \\
\lambda_{1} \cos \gamma \sin \theta\left(\lambda_{1}-1\right) \sin 2 \beta
\end{array}\right\}
$$


Eq. (9) now contains only one unknown coefficient $A_{1}$ which can be measured using single strain gage. It can be conveyed here that gage line is the line, making an angle $\theta$ with the notch axis, on which strain gage is pasted in the direction of $\beta$ at a radial distance $r$ from the tip of the notch (Fig. 2). Therefore, by placing one strain gage at a point defined by $P(r, \theta)$ along the gage line and orienting it at an angle of $\beta$ with respect to the notch axis, the mode I NSIF $K_{I}^{V}$ can be conveniently determined using Eq. (9) and Eq. (5). It is to be noted that although Eq. (9) comprises only one coefficient, yet the equation on the right hand side is actually a three parameter representation of $\varepsilon_{a}$ as expressed in the Eq. (8). Consequently, strain gage can be placed at longer radial distances with respect to the notch tip and this would reduce various effects arising in the strain gage technique. Determination of $\theta$ and $\beta$ for a given Poisson's ratio $v$ is done through a systematic approach by eliminating the functions $f_{2}$ and $f_{2}^{*}$ (Eq. (8)) i.e. by equating these functions to zero. In case of $\gamma \leq 40^{\circ}$, only the function $f_{2}$ exists due to the existence of real eigen value and real Williams coefficient. Hence, in such case, there will be only one equation if the function $f_{2}$ is equated to zero, thus leading to infinitely many solutions for $\theta$ and $\beta$. In case of $\gamma>40^{\circ}$, the functions $f_{2}$ and $f_{2}^{*}$ will co-exist due to the complex nature of eigen value and corresponding Williams coefficient. In such case, there will be two equations if the functions $f_{2}$ and $f_{2}^{*}$ are simultaneously equated to zero., thus leading to a unique set of $\theta$ and $\beta$. All these as discussed can be followed from the published work of Paul et al. [23].

\subsection{Assessment of the valid or optimal radial strain gage locations}

The Eq. (9) clearly shows that prior estimation of valid or optimal radial location of the strain gage is essential for the appropriate determination of NSIF $K_{I}^{V}$. Interestingly, the published work of Paul et al. [23] exhibits the theoretical basis for obtaining valid or optimal radial strain gage locations. These locations are optimal in the sense that they can be selected in a range as follows

$$
r_{\min } \leq r \leq r_{\max }
$$

where

$$
r_{\min }=1.25 \times \text { thickness of the plate }
$$

Thus, any radial strain gage location at distance $r$ from the notch tip along the gage line if satisfies the criterion of Eq. (11) should be understood as a valid or optimal radial strain gage location. Eq. (11) helps to ensure that the gages are not pasted beyond $r_{\max }$, however the gage reading is expected to obey Eq. (9). The procedure to determine the maximum permissible radial strain gage distance $r_{\max }$ can be obtained in details from the published work as given in Ref.[23]. However, it can be briefly mentioned here that the value of $r_{\max }$ is obtained from the plot of $\ln \left(\varepsilon_{a}\right)$ versus $\ln (r)$ and the plot of $\ln \left(\varepsilon_{a}\right)$ versus $\ln (r)$ is achieved through finite element simulation.

\section{Results and discussions}

It is studied from the preceding sections that determining valid or optimal radial strain gage locations a priori is very much essential for obtaining NSIF of a specific two-dimensional sharp V-notched body. A hint has been given above with appropriate mathematical equations for showing the procedure to determine the valid or optimal radial strain gage locations. In the published work of Paul et al. [23], it can be observed that the maximum permissible radial strain gage distance $r_{\max }$ varies with notch length to width ratio $a / b$. For a given notch angle $\gamma$, the $r_{\max }$ value increases as $a / b$ increases from 0.2 to 0.4 , however it starts decreasing as $a / b$ increases further from 0.4 to 0.8 . Furthermore, it is also observed that the $r_{\max }$ value increases when notch angle $\gamma$ increases for a given notch length to width ratio $a / b$. All these observations [23] were found for a particular material with $E=2917 \mathrm{MPa}$ and $v=0.382$. However, it is crucial to know whether material properties play any role in such graphical trends of $r_{\max }$ or not. For this study, a similar problem is accomplished with a different material having $E=200 \mathrm{GPa}$ and Determamatithe results are subsequently presented in this conferendo paper. Sharp V-notched SENT configurations are again considered for the present analysis as same as Ref.[23]. Finite element simulations have been carried out to determine the maximum permissible radial strain gage distance $r_{\max }$. A typical FEA mesh (Fig.3) which is used for the finite element simulation can be seen below

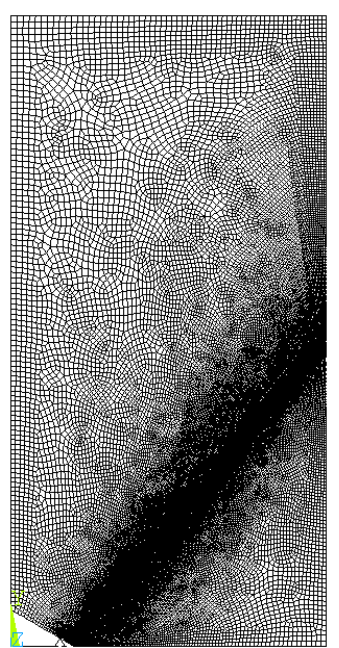


Fig.3. A typical FEA mesh in its finest state with the inclined darkened portion representing the strain gage line along which the strain gage is placed at certain optimal or valid location

The geometric, material and loading parameters associated with the problem of sharp V-notched (SENT) configurations subjected to mode I loading under plane stress condition are presented in Table 1 and Table 2 respectively.

Table 1 Geometric, loading and material parameters for notch angles $30^{\circ}$ and $60^{\circ}$ respectively

\begin{tabular}{ccccccc}
\hline $\begin{array}{c}\text { Notch } \\
\text { angle } \\
(\gamma)\end{array}$ & $\begin{array}{c}\text { First } \\
\text { eigen } \\
\text { value } \\
\left(\lambda_{1}^{\prime}\right)\end{array}$ & $\begin{array}{c}b \\
(\mathrm{~mm})\end{array}$ & $h / b$ & $\begin{array}{c}E \\
(\mathrm{GPa})\end{array}$ & $v$ & $\begin{array}{c}\sigma \\
(\mathrm{MPa})\end{array}$ \\
& & & & & \\
\hline $30^{\circ}$ & 0.5015 & 150 & 2 & 200 & $1 / 3$ & 100 \\
$60^{\circ}$ & 0.5122 & 150 & 2 & 200 & $1 / 3$ & 100 \\
\hline
\end{tabular}

Table 2 Gage line orientation $\theta$ and strain gage orientation $\beta$ for the notch angles $30^{\circ}$ and $60^{\circ}$ respectively

\begin{tabular}{ccc}
\hline $\begin{array}{c}\text { Notch } \\
\text { angle } \\
(\gamma)\end{array}$ & $\begin{array}{c}\text { Gage line } \\
\text { orinetation } \\
(\theta)\end{array}$ & $\begin{array}{c}\text { Stran gage } \\
\text { orinetation } \\
(\beta)\end{array}$ \\
\hline $30^{\circ}$ & $50.49^{\circ}$ & $52.54^{\circ}$ \\
$60^{\circ}$ & $50.93^{\circ}$ & $44.50^{\circ}$ \\
\hline
\end{tabular}

It was already discussed in preceding sections that the maximum permissible radial strain gage distance $r_{\max }$ value is usually obtained from the plot of $\ln \left(\varepsilon_{a}\right)$ versus $\ln (r)$ and the plot of $\ln \left(\varepsilon_{a}\right)$ versus $\ln (r)$ is obtained through finite element simulation. A straight line having a negative slope has to be superimposed on the plot of $\ln \left(\varepsilon_{a}\right)$ versus $\ln (r)$. The point at which the straight line representing the ideal solution deviates from the plot of $\ln \left(\varepsilon_{a}\right)$ versus $\ln (r)$ representing the actual solution obtained from finite element simulation would give us the $r_{\max }$ value as shown in Fig.4. The graph in Fig.4 needs to be observed from right hand side to left hand side to obtain the point of deviation. While observing from right hand side to left hand side of the graph (Fig.4), if the percentage relative error between the ideal solution and the finite element solution is found to be falling just below $0.5 \%$ then the point of deviation is said to be reached. The schematic as shown in Fig.4 can be regarded as the usual way of obtaining the $r_{\max }$ value. The plots (Fig.4) are obtained for the problem presented in this section for notch angle $\gamma=60^{\circ}$. It can be concluded from Fig. 4 that for a specific notch angle the $r_{\max }$ value rises when $a / b$ increases from 0.2 to 0.4 but the same dips when $a / b$ increases further from 0.4 to 0.8 . It is one of the major observations. Exactly similar observation was also made in Ref.[23] with a different material .

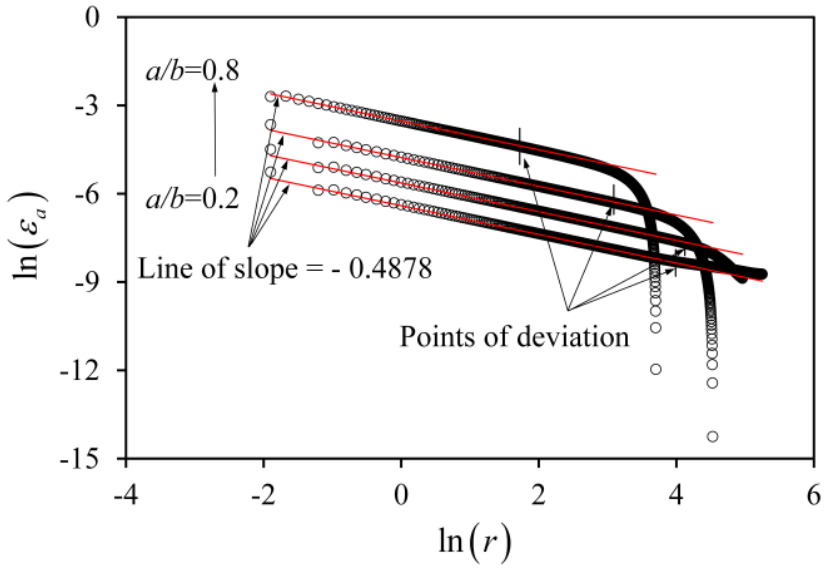

Fig.4. Variations of $\ln \left(\varepsilon_{a}\right)$ with $\ln (r)$ along the gage line for different $a / b$ ratio for the SENT configuration with the notch angle $\gamma=60^{\circ}$.

Another important observation is made from Fig.5. Fig.5 shows that the trend of obtaining $r_{\max }$ value with $a / b$ varying from 0.2 to 0.8 remains consistent for a completely different notch angle also. In addition, it is also observed from Fig.5 that as the notch angle increases, the $r_{\max }$ value also increases for a specific $a / b$ ratio. Exactly similar observation (Fig.5) was also made in Ref.[23] with a different material . Table 3 presents the numerical results (the $r_{\max }$ values) in support of the above three important observations thus made.

Table 3 Values of $r_{\max }$ for different $a / b$ ratio from 0.2 to 0.8 and for notch angles $30^{\circ}$ and $60^{\circ}$ respectively

\begin{tabular}{cccc}
\hline $\begin{array}{c}\text { Notch angle } \\
(\gamma)\end{array}$ & $a / b$ & $r_{\max }(\mathrm{mm})$ & $r_{\max } / b$ \\
\hline $30^{\circ}$ & 0.2 & 17.9347 & 0.119564667 \\
& 0.4 & 50.6119 & 0.337412667 \\
& 0.6 & 9.0448 & 0.060298667 \\
& 0.8 & 3.5155 & 0.023436667 \\
$60^{\circ}$ & 0.2 & 54.0228 & 0.360152 \\
& 0.4 & 61.7558 & 0.411705333 \\
& 0.6 & 21.9829 & 0.146552667 \\
& 0.8 & 5.5998 & 0.037332 \\
\hline
\end{tabular}




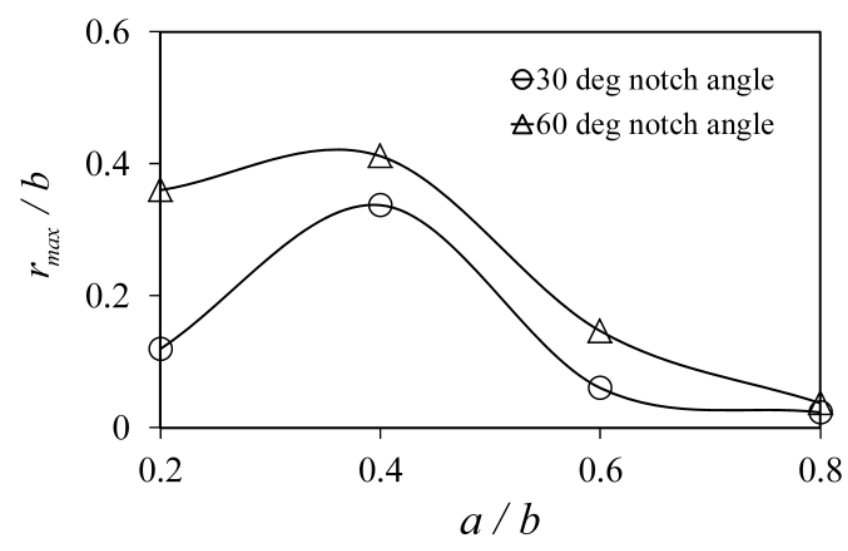

Fig.5. Variation of $r_{\max } / b$ with $a / b$ for the SENT configurations for notch angles $30^{\circ}$ and $60^{\circ}$ respectively

\section{Conclusions}

As seen from the results section of this paper, even after changing the material, the graphical trends of the $r_{\max }$ values remain consistent as same as reported in Ref. [23]. This is a crucial observation presented in this paper. Material independence of the graphical trends is a completely novel finding and it is no where published earlier. However, the $r_{\max }$ values of course changed with different material. The graphical trends which we have discussed all about in the results section can be summarised as : for a specific notch angle the $r_{\max }$ value rises when $a / b$ increases from 0.2 to 0.4 but the same dips when $a / b$ increases further from 0.4 to 0.8 , such trend remains consistent for different notch angles and the $r_{\max }$ value increases for a specific $a / b$ ratio as the notch angle increases.

\section{References}

1. M.L. Dunn, W. Suwito, S. Cunningham, Eng. Fract. Mech., 57, 417-430 (1997).

2. K. Tomari, S. Tonogai, T. Harada, H. Hamada, K. Lee, T. Morii, Z. Maekawa, Polym. Eng. Sci., 30, 931-936 (1990).

3. M.L. Williams, J. Appl. Mech.,19, 526528(1952).

4. M.L. Dunn, W. Suwito, S. Cunningham, Int. J. Solids. Struct., 34, 3873-3883 (1997).

5. M.L. Dunn, W. Suwito, S. Cunningham, C.W. May, Int. J. Fracture, 84, 367-381 (1997).

6. A. Seweryn, Eng. Fract. Mech., 47, 673-681 (1994).

7. A. Carpinteri, Eng. Fract. Mech., 26,143-155 (1987).

8. Z. Yosibash, E. Priel, D. Leguillon, Int. J. Fracture, 141, 291-312 (2006).
9. S.Q. Hou, J.Q. Xu, Int. J. Mech. Sci., 88, 202207 (2014).

10. Z. Yosibash, A.Bussiba, I. Gilad, Int. J. Fracture, 125, 307-333 (2004).

11. P. Lazzarin, R. Zambardi, Int. J. Fracture, 112, 275-298 (2001).

12. M.P. Savruk, A. Kazberuk, Int. J. Fracture, 161, 79-95 (2010).

13. M. Stern, E.B. Becker, R.S. Dunham, Int. J. Fracture, 12, 359-368 (1976).

14. M.R. Ayatollahi, M. Nejati, Int.J. Mech. Sci., 53, 164-177 (2011).

15. B. Gross, A. Mendelson, Int. J. Fract. Mech., 8, 267-276 (1972).

16. T. Yu, L. Shi, J. Strain. Anal. Eng., 47, 95-103 (2012).

17. T. Kondo, M. Kobayashi, H. Sekine, Exp. Mech., 41, 1-7 (2001).

18. T. Kondo, Y. Kurabe, T. Sasaki, T. Kurahashi, Y. Miyashita, Eng. Fract. Mech., 124-125, 248261 (2014).

19. M.R. Ayatollahi, M. Nejati, Mater. Design, 32, 561-569 (2011).

20. J.N.Prassianakis, P.S. Theocaris, J. Phys. D: Appl. Phys., 13, 1043-1053 (1980).

21. X.F. Yao, H.Y. Yeh, W. Xu, Int. J. Solids Struct., 43, 1189-1200 (2006).

22. M.R.Y. Dehnavi, I. Eshraghi, N. Soltani, Polym. Test., 32, 778-784 (2013).

23. P.Paul, K.S.R.K.Murthy, D.Chakraborty, Theor. Appl. Fract. Mec., 94, 57-70 (2018). 